\title{
Asymmetrical Normal-Zone Propagation Observed in the Aluminum-Stabilized Superconductor for the LHD Helical Coils
}

Nagato Yanagi, Shinsaku Imagawa, Yoshimitsu Hishinuma, Kazutaka Seo, Kazuya Takahata, Shinji Hamaguchi, Akifumi Iwamoto, Hirotaka Chikaraishi, Hitoshi Tamura, Sadatomo Moriuchi, Shuichi Yamada, Arata Nishimura, Toshiyuki Mito, and Osamu Motojima

\begin{abstract}
Transient normal-transitions have been observed in the superconducting helical coils of the Large Helical Device (LHD). Stability tests have been performed for an $R \& D$ coil as an upgrading program of LHD, and we observed asymmetrical propagation of an initiated normal-zone. In some conditions, a normal-zone propagates only in one direction along the conductor and it hence forms a traveling normal-zone. The Hall electric field generated in the longitudinal direction in the aluminum stabilizer is a plausible candidate to explain the observed asymmetrical normal-zone propagation.
\end{abstract}

Index Terms-Aluminum stabilizer, dynamic stability, helical coils, LHD, longitudinal Hall electric field, traveling normal-zone.

\section{INTRODUCTION}

D URING the last six experimental cycles of the Large Helical Device (LHD), which is performing reactor-extrapolative high temperature plasma experiments with a heliotron confinement approach [1], excitation tests of the superconducting coil system have been systematically carried out. It has been observed that the pool-cooled helical coils (major radius $3.9 \mathrm{~m}$, minor radius $\sim 1 \mathrm{~m}$ and toroidal pitch number 10) allow transient normal-transitions at a current and magnetic field slightly lower than the specified operation point (13 kA and $6.9 \mathrm{~T}$ at temperature $4.4 \mathrm{~K}$ ) [2], [3].

For the helical windings, a composite-type superconductor is used, which consists of a NbTi/Cu Rutherford cable, a pure aluminum stabilizer and a copper sheath. One of the most important features of this superconductor is that $\mathrm{Cu}-2 \% \mathrm{Ni}$ is used as the clad material around the pure aluminum stabilizer to effectively reduce the Hall current generation and hence improve the cryogenic stability [4].

The characteristics of this superconductor have been intensively studied by carrying out short sample tests as well as a small R\&D coil test. It was confirmed that the conductor becomes transiently unstable even when the transport current is lower than the steady-state "cold-end" recovery current, and an initiated normal-zone temporarily propagates over a finite length. It should be noted that the magnetic (current) diffusion process in the pure aluminum stabilizer is relatively slow due to its low resistivity. Therefore, the effective longitudinal resis-

Manuscript received October 21, 2003.

The authors are with the National Institute for Fusion Science, 322-6 Oroshi-cho, Toki, Gifu 509-5292, Japan (e-mail: yanagi@nifs.ac.jp).

Digital Object Identifier 10.1109/TASC.2004.830673

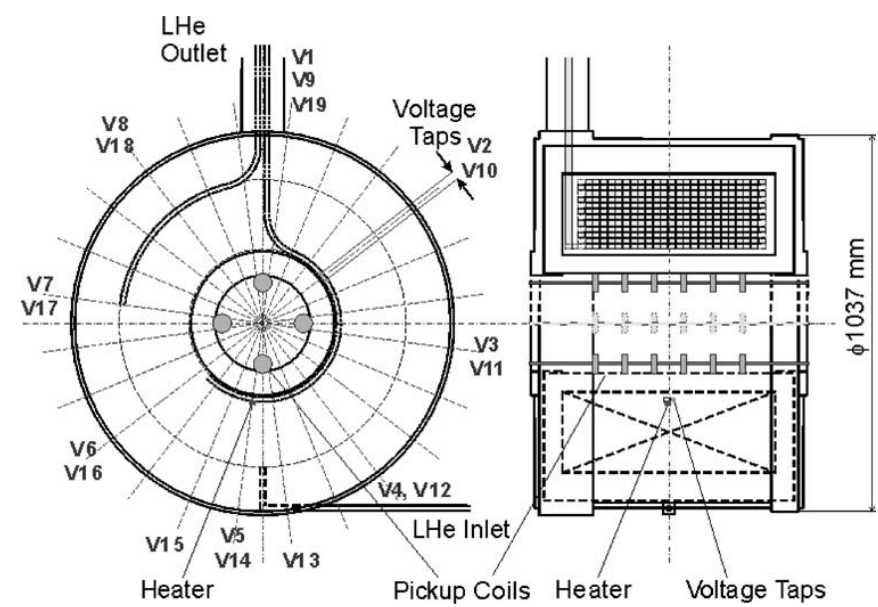

Fig. 1. Side and cross-sectional views of the R\&D coil. Positions of a resistive heater, voltage taps and pick-up coils are schematically illustrated. The typical length of a voltage tap $(23 \mathrm{~mm})$ is indicated for the voltage taps of V2 and V10.

tance of the stabilizer remains considerably higher than that in the steady state during the characteristic diffusion time, and it might cause a transient degradation of the cryogenic stability [5].

In the series of conductor tests, it was confirmed that the propagation velocity of the generated normal-zone shows asymmetry with respect to the two directions along the conductor when the external magnetic field was applied parallel to the wider surface of the conductor [6]. On the other hand, there was practically no difference between the two propagation velocities when the magnetic field was applied in the perpendicular direction. We should also note that for the former case, we even observed a one-side propagation of an initiated normal-zone in some conditions.

\section{EXPERIMENTAL OBSERVATIONS OF ASYMMETRICAL} NORMAL-ZONE PROPAGATION IN THE SUB-COOLED R\&D COIL

One method to improve the cryogenic stability of a poolcooled superconducting coil is to lower the operating temperature by supplying sub-cooled liquid helium. In order to examine this effect, an R\&D coil was fabricated using the same superconductor as that for the LHD helical coils [7]. The inner and outer radii of the windings are 200 and $777 \mathrm{~mm}$, respectively, and a side and cross-sectional views of the coil are shown in Fig. 1. It was designed to match the load line of that of the LHD helical 


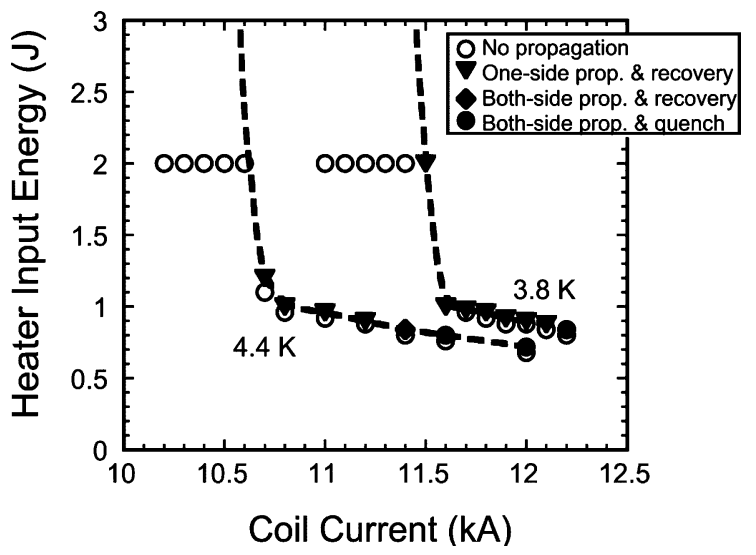

Fig. 2. Heater input energy as a function of the coil current.

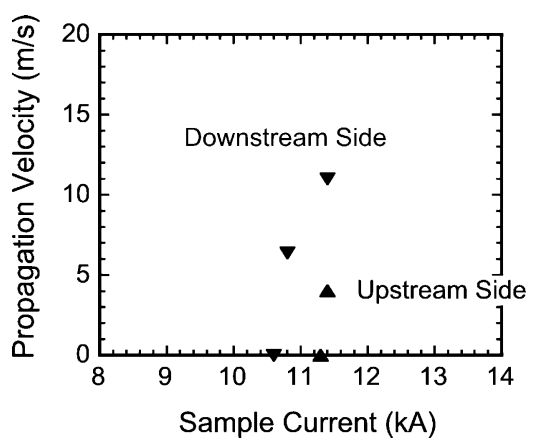

Fig. 3. Dependence of the propagation velocity of the normal-zone on the coil current (temperature: $4.4 \mathrm{~K}$ ).

coils, i.e., the maximum magnetic field at the innermost windings is $6.9 \mathrm{~T}$ with the coil current of $13.0 \mathrm{kA}$. The $\mathrm{R} \& \mathrm{D}$ coil was situated in a cryostat that has two-staged cryogenic compressors and a heat exchanger. The coil was tested first with saturated liquid helium (temperature: $4.4 \mathrm{~K}$ ), and then sub-cooled liquid helium of 3.1-4.2 K was supplied.

In the R\&D coil test, the cryogenic stability was examined using a resistive heater attached to the surface of the innermost conductor. Fig. 2 shows the input energy of the heater required for initiating a normal-transition as a function of the coil current for two temperature cases. As is seen in Fig. 2, the minimum propagating current could be improved from $10.7 \mathrm{kA}$ at $4.4 \mathrm{~K}$ to $11.6 \mathrm{kA}$ at $3.8 \mathrm{~K}$.

Normal-zone propagation was monitored using voltage taps distributed along the innermost windings. Fig. 3 shows the dependence of the measured propagation velocity on the coil current. It is clearly seen that the propagation velocity is much faster in the downstream side of the transport current than that in the upstream side, and it hence reveals an asymmetry of normal-zone propagation, as was observed in the previous conductor tests. It should be particularly noted that in the current region of 10.7 to $11.4 \mathrm{kA}$, the normal-zone propagates only in the downstream side, which is also the same phenomenon as that was observed in short sample conductors [6], a small R\&D coil [3] and the LHD helical coils [8].

Fig. 4 plots the spatial profiles of the measured longitudinal resistance by taking the time after the turn-on of the heater pulse as a parameter. With the coil current of $11.4 \mathrm{kA}$, the normal-zone propagates in two directions, the spatial profiles, however, show
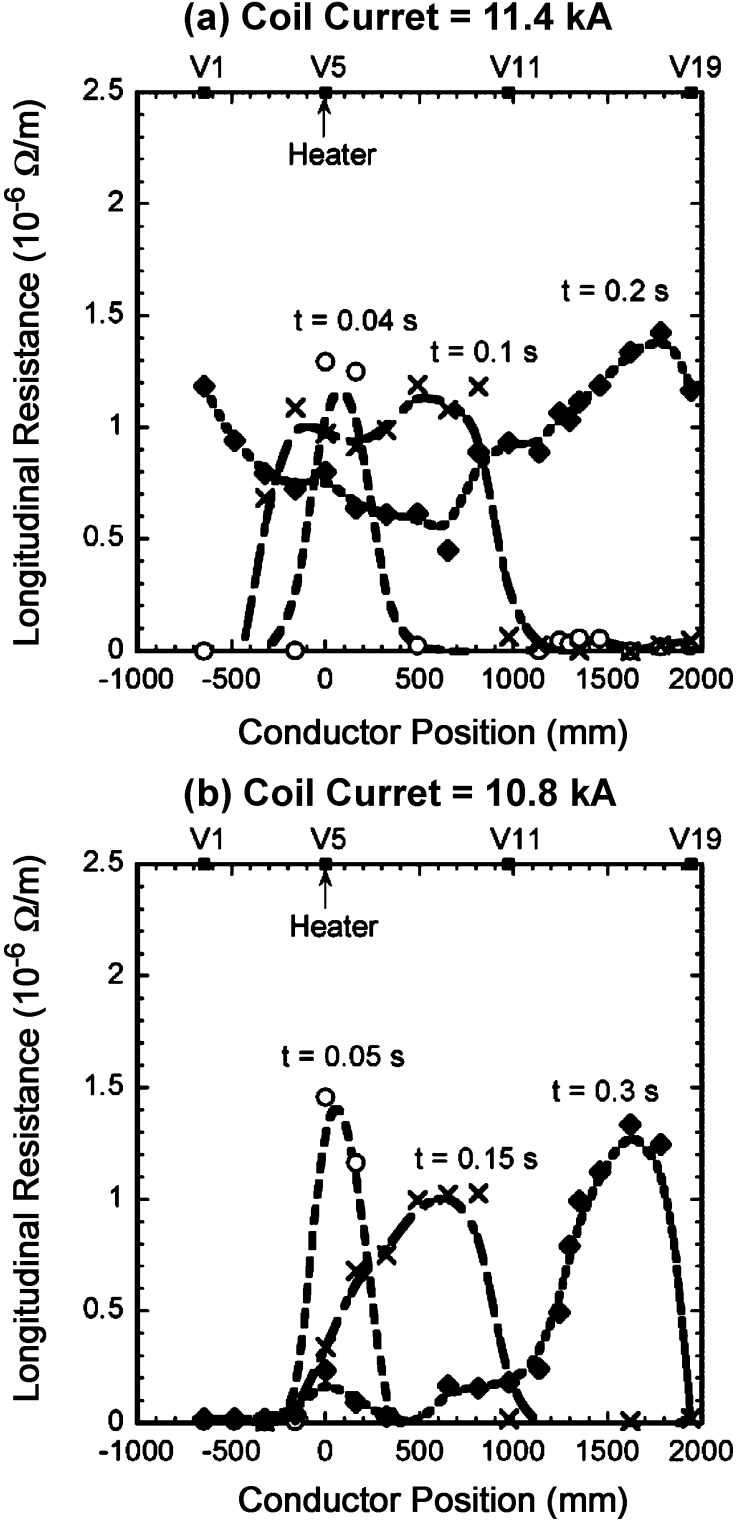

Fig. 4. Spatial profiles of the observed normal-zones with the coil current of (a) $11.4 \mathrm{kA}$ and (b) $10.8 \mathrm{kA}$, both at temperature $4.4 \mathrm{~K}$.

asymmetry as the propagation velocities differ. On the other hand, in the case of a lower current of $10.8 \mathrm{kA}$, the normal-zone propagates only in one direction, which is the downstream side of the transport current, and the spatial profile of the longitudinal resistance clearly shows a form of "traveling normalzone" which was previously observed for another type of aluminum-stabilized superconductor developed for a SMES application [9].

\section{Plausible Mechanism for ExPlaining ThE ASYMMETRICAL NORMAL-ZONE PROPAGATION}

\section{A. Model with Longitudinal Hall Electric Field}

The observed asymmetry in the propagation velocity of the normal-zone can be explained if we assume some difference in the current transferring process occurring at two normal-tosuperconducting (n-s) boundaries. One plausible candidate for causing such a difference is the Hall electric field generated in 


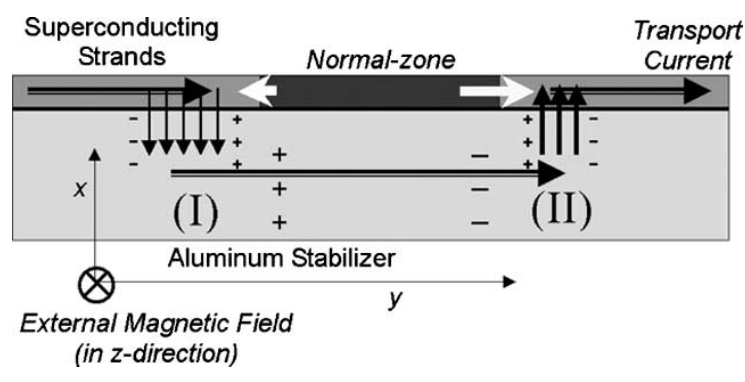

Fig. 5. Schematic illustration of the current transferring process from the superconducting strands to the aluminum stabilizer.

the aluminum stabilizer in the longitudinal direction. The corresponding model is schematically illustrated in Fig. 5. Here we assume that the current transfer occurs from the superconducting strands to the aluminum stabilizer by perpendicularly crossing the external magnetic field, which corresponds to the present case.

We now assume that the conductor is along the $y$-direction and the external magnetic field $B$ is applied to the $z$-direction. When a normal-transition occurs at some point, the electric field is developed in the longitudinal direction in the stabilizer by satisfying the following relation

$$
E_{y}=\rho j_{y}+R_{H} B j_{x} .
$$

Here, $\rho$ is the resistivity, $j$ is the current density and $R_{H}$ is the Hall coefficient. For pure aluminum, $R_{H}$ is given approximately as $1.0 \times 10^{-10} \Omega \mathrm{m} / \mathrm{T}$. Near the $\mathrm{n}$-s boundaries, the transport current is transferred from the strands to the stabilizer in the $x$-direction, which means that $j_{x}$ becomes finite and the second term in (1) should be considered. Here we should note that this term changes its sign in the two n-s boundaries with the configuration illustrated in Fig. 5. The additional electric field given by this process possibly leads to a change of the effective longitudinal resistance of the stabilizer, and hence the characteristic length of the current transferring process. Thus, the heat generation in the vicinity of two n-s boundaries should differ and the propagation velocity might resultantly be changed.

\section{B. Evaluation of the Characteristic Length of Current Transfer and Joule-Heating Power}

Now we analytically solve the process of current transfer based on a one-dimensional analysis. The transport current is assumed to flow in the positive direction of $y$. The function $I(y)$ is defined as the total current flowing in the stabilizer at position $y$. The potential $\phi(y)$ is determined in the stabilizer, whereas the potential should be zero in the superconducting strands. We assume that there is a thin resistive layer between the superconducting strands and the stabilizer, as is shown in Fig. 5, and it gives a contact resistance of $r$ per unit length. The longitudinal resistance of the stabilizer is given as $R$ per unit length. Then, the basic equations governing the current transferring process are given by modifying the equations given in [10] as

$$
\begin{aligned}
\phi(y) & =-r \frac{d I(y)}{d y} \\
\frac{d \phi(y)}{d y} & =-I(y) R+\frac{d I(y)}{d y} \frac{R_{H} B}{L} .
\end{aligned}
$$

Here, the conducting materials are all assumed to have the width $L$ and the last term in (3) corresponds to the longitudinal Hall electric field. We then assume that a normal-zone exists in the region defined by $y \geq 0$ and the left side of this region $(y<0)$ is in the superconducting state. Here we note that this situation corresponds to the n-s boundary indicated by (I) in Fig. 5. We also assume that the current $I(y)$ can be expressed by the following form [11]

$$
I(y)=\left\{\begin{array}{ll}
I_{0} e^{\lambda y} & (y<0) \\
I_{0} & (y \geq 0)
\end{array} .\right.
$$

A simple calculation gives the characteristic length of current transfer at the n-s boundary (I) as

$$
\xi_{1}=\frac{1}{\lambda}=\frac{2 r}{-\beta+\sqrt{\beta^{2}+4 r R}},
$$

where $\beta=R_{H} B / L$. The same derivation gives the characteristic length for the other n-s boundary (II) as

$$
\xi_{2}=\frac{2 r}{\beta+\sqrt{\beta^{2}+4 r R}} .
$$

If these values are compared with the characteristic length of $\xi_{0}=\sqrt{r / R}$ for zero magnetic field, or without including the longitudinal Hall electric field, we easily observe the relation $\xi_{1}>\xi_{0}>\xi_{2}$. This means that the characteristic length of current transfer b ecomes longer at n-s boundary (I), whereas it is shorter at (II). This is caused by the mechanism that the longitudinal electric field is reduced from the intrinsic value at (I), whereas it is enhanced at (II). Applying the physical parameters for the superconductor of the LHD helical coils, $\xi_{1}=75 \mathrm{~mm}$ and $\xi_{2}=14 \mathrm{~mm}$ are obtained, which should be compared with $\xi_{0}=32 \mathrm{~mm}$. Here, the contact resistance $r$ is evaluated by the resistivity and thickness of the $\mathrm{CuNi}$ layer and the resistance of the aluminum stabilizer $R$ is given by its effective value by considering the Hall current generation in the transverse direction [4], [5].

From the equations derived above, we now obtain the joule heating power per unit length as

$$
P(y)=I_{0}^{2} e^{2 \lambda y}\left(R+\lambda \beta+r \lambda^{2}\right) .
$$

The integration of $P(y)$ in the superconducting region gives the total joule heating power with respect to the current transferring process as

$$
P_{i}=\frac{I_{0}^{2} r}{\xi_{i}} \quad(i=1,2) .
$$

Equation (5) means that the total heat generation at n-s boundary (II) is about five times higher than that at (I) for the present conductor as the characteristic length of current transfer becomes shorter at (II). Fig. 6 shows the distribution of $P(y)$ at the two n-s boundaries. We observe that the peak power density at (II) is about thirty times higher than that at (I). We consider that this feature seems to explain the observed asymmetrical normal-zone propagation. 


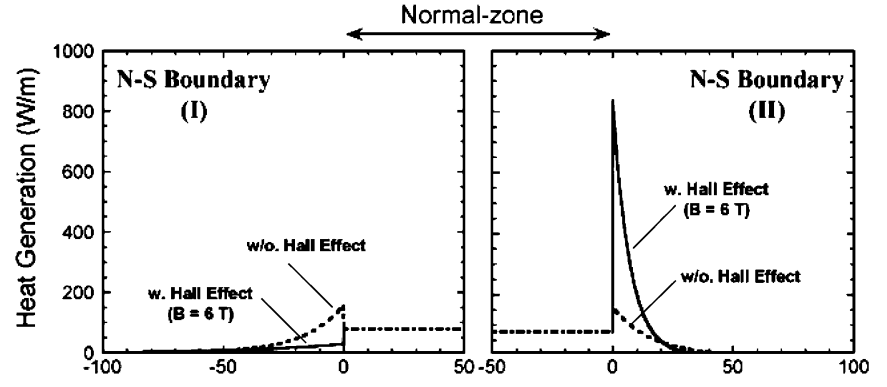

Fig. 6. Calculated longitudinal distribution of the joule-heating power at the two current transfer regions. The transport current is $10 \mathrm{kA}$ and the external magnetic field is $6 \mathrm{~T}$. The bold lines correspond to the heat generation by including the longitudinal Hall electric field using (4), whereas, the dashed lines are without this effect.

\section{DISCUSSION}

In the above one-dimensional analysis, the characteristic length of current transfer is derived for the steady-state condition. However, in reality, an initiated normal-zone propagates in time and the whole process should be treated as a transient problem. One important point is that the magnetic (current) diffusion process in the pure aluminum stabilizer is rather slow compared to the characteristic time of normal-zone propagation. For the present conductor, the diffusion time in the aluminum stabilizer is estimated as $50 \mathrm{~ms}$ [6], and within this period, the normal-zone front moves over $250 \mathrm{~mm}$ with the propagation velocity of $5 \mathrm{~m} / \mathrm{s}$. This length is much longer than the characteristic length of current transfer given in the above steady-state problem. Thus, the real situation should be treated in a moving coordinate and both the contact resistance $r$ and the longitudinal resistance $R$ should be replaced by their effective values.

Another crucial point for more accurately discussing the present problem is that the current is transferred also into the copper sheath within a short time scale (compared to the magnetic diffusion process in the aluminum) near the $n-s$ boundaries. For a slab geometry in which a pure aluminum is placed in one side of a flat superconducting layer and a copper in the other side, the exact analytical solutions for giving the current densities in the aluminum and copper can be obtained by simultaneously solving the magnetic diffusion processes in the two electrical conducting media. The result shows that about $30 \%$ of the transport current initially flows into the copper sheath, and therefore, the current transferring process should be properly treated also in this part. Here, we should note that though the Hall coefficient of copper $(\sim-0.55 \times \Omega \mathrm{m} / \mathrm{T})$ has the opposite sign, the direction of current transfer, i.e., the sign of $j_{x}$, also becomes opposite, and thus the longitudinal Hall electric field is generated in the same direction as that for aluminum. In this connection, we also consider that the present effect should be observed in any conductors (not only for aluminum-stabilized ones) provided they have asymmetrical configurations.

A more accurate analysis should be done by simultaneously including the current transferring process from the superconducting strands to the aluminum and copper in a moving coordinate as well as the current diffusion process in these stabilizers. This might be only possible by numerical simulations and it will be our future work.

\section{SUMMARY}

Asymmetrical normal-zone propagation was observed in the R\&D coil test for the upgrading program of the LHD helical coils. In some conditions, the generated normal-zone propagates only in one direction along the conductor and it hence forms a traveling normal-zone. The Hall electric field generated in the longitudinal direction in the stabilizers might be effective to alter the characteristic length of current transfer in two n-s boundaries and hence cause the asymmetrical propagation of the normal-zone.

\section{REFERENCES}

[1] A. Iiyoshi, A. Komori, and A. Ejiri et al., "Overview of the Large Helical Device project," Nuclear Fusion, vol. 39, pp. 1245-1256, 1999.

[2] S. Imagawa, N. Yanagi, and T. Mito et al., "Excitation properties and cryogenic stability of helical coils for the LHD," IEEE Trans. Appl. Supercond., vol. 11, no. 1, pp. 1889-1892, March 2001.

[3] N. Yanagi, S. Imagawa, and A. V. Gavrilin et al., "Analysis on the normal transition event of the LHD helical coils," IEEE Trans. Appl. Supercond., vol. 10, no. 1, pp. 610-613, March 2000.

[4] H. Kaneko and N. Yanagi, "Enhancement of magneto-resistance due to Hall current in aluminum-copper composite," Cryogenics, vol. 32, pp. 1114-1120, 1992.

[5] A. V. Gavrilin, N. Yanagi, S. Satoh, and O. Motojima, "Computer simulation of normal zone propagation in the LHD helical coils," Advances in Supercond., vol. 11, no. 1, pp. 1447-1450, March 1999.

[6] N. Yanagi, A. V. Gavrilin, and T. Mito et al., "Stability characteristics of the aluminum stabilized superconductor for the LHD helical coils," Advances in Supercond., vol. 11, no. 1, pp. 991-994, 1999.

[7] S. Imagawa, N. Yanagi, and Y. Hishinuma et al., "Results of Stability Test in Sub-Cooled Helium for the R\&D of the LHD Helical Coil,", this conference.

[8] S. Imagawa, N. Yanagi, and H. Sekiguchi et al., "Performance of the Helical Coils for the Large Helical Device in Six Years' Operation,", this conference.

[9] J. M. Pfotenhauer, M. K. Abdelsalam, and F. Bodker et al., "Test results from SMES proof of principle experiment," IEEE Trans Magn., vol. 27, pp. 1704-1707, 1991.

[10] M. N. Wilson, Superconducting Magnets: Oxford University Press, 1983, pp. 233-235.

[11] H. Kaneko, "Insulation of highly conductive metal in composite stabilizer for reduction of Hall current across surface," Cryogenics, vol. 33, pp. 1077-1085, 1993. 